\title{
Real-time independent component analysis of fMRI time-series
}

Citation for published version (APA):

Esposito, F., Seifritz, E., Formisano, E., Morrone, R., Scarabino, T., Cirillo, S., Goebel, R., \& Di Salle, F. (2003). Real-time independent component analysis of fMRI time-series. Neuroimage, 20(4), 2209-2224. https://doi.org/10.1016/j.neuroimage.2003.08.012

Document status and date:

Published: 01/01/2003

DOI:

10.1016/j.neuroimage.2003.08.012

Document Version:

Publisher's PDF, also known as Version of record

Document license:

Taverne

Please check the document version of this publication:

- A submitted manuscript is the version of the article upon submission and before peer-review. There can be important differences between the submitted version and the official published version of record.

People interested in the research are advised to contact the author for the final version of the publication, or visit the DOI to the publisher's website.

- The final author version and the galley proof are versions of the publication after peer review.

- The final published version features the final layout of the paper including the volume, issue and page numbers.

Link to publication

\footnotetext{
General rights rights.

- You may freely distribute the URL identifying the publication in the public portal. please follow below link for the End User Agreement:

www.umlib.nl/taverne-license

Take down policy

If you believe that this document breaches copyright please contact us at:

repository@maastrichtuniversity.nl

providing details and we will investigate your claim.
}

Copyright and moral rights for the publications made accessible in the public portal are retained by the authors and/or other copyright owners and it is a condition of accessing publications that users recognise and abide by the legal requirements associated with these

- Users may download and print one copy of any publication from the public portal for the purpose of private study or research.

- You may not further distribute the material or use it for any profit-making activity or commercial gain

If the publication is distributed under the terms of Article $25 \mathrm{fa}$ of the Dutch Copyright Act, indicated by the "Taverne" license above, 


\title{
Real-time independent component analysis of fMRI time-series
}

\author{
Fabrizio Esposito, ${ }^{\mathrm{a}, \mathrm{e}, \mathrm{g}, *}$ Erich Seifritz, ${ }^{\mathrm{b}}$ Elia Formisano, ${ }^{\mathrm{c}}$ Renato Morrone, ${ }^{\mathrm{d}}$ \\ Tommaso Scarabino, ${ }^{\mathrm{e}}$ Gioacchino Tedeschi, ${ }^{\mathrm{a}, \mathrm{e}}$ Sossio Cirillo, ${ }^{\mathrm{f}}$ \\ Rainer Goebel, ${ }^{\mathrm{c}}$ and Francesco Di Salle ${ }^{\mathrm{e}, \mathrm{g}}$ \\ a Second Division of Neurology, Second University of Naples, 80138 Naples, Italy \\ ${ }^{\mathrm{b}}$ Department of Psychiatry, University of Basel, 4025 Basel, Switzerland \\ c Department of Cognitive Neuroscience, Maastricht University, 6200 MD Maastricht, The Netherlands \\ d "Morrone" Diagnostic Center, 81100 Caserta, Italy \\ e Department of Neuroradiology, Scientific Institute “Casa Sollievo della Sofferenza”, 71013 S. Giovanni Rotondo, Italy \\ ${ }^{\mathrm{f}}$ Department of Neuroradiology, Second University of Naples, 80138 Naples, Italy \\ g Department of Neurological Sciences, University of Naples "Federico II", 80131 Naples, Italy
}

Received 4 March 2003; revised 9 August 2003; accepted 14 August 2003

\begin{abstract}
Real-time functional magnetic resonance imaging (fMRI) enables one to monitor a subject's brain activity during an ongoing session. The availability of online information about brain activity is essential for developing and refining interactive fMRI paradigms in research and clinical trials and for neurofeedback applications. Data analysis for real-time fMRI has traditionally been based on hypothesis-driven processing methods. Off-line data analysis, conversely, may be usefully complemented by data-driven approaches, such as independent component analysis (ICA), which can identify brain activity without a priori temporal assumptions on brain activity. However, ICA is commonly considered a time-consuming procedure and thus unsuitable to process the high flux of fMRI data while they are acquired. Here, by specific choices regarding the implementation, we exported the ICA framework and implemented it into real-time fMRI data analysis. We show that, reducing the ICA input to a few points within a time-series in a sliding-window approach, computational times become compatible with real-time settings. Our technique produced accurate dynamic readouts of brain activity as well as a precise spatiotemporal history of quasistationary patterns in the form of cumulative activation maps and time courses. Results from real and simulated motor activation data show comparable performances for the proposed ICA implementation and standard linear regression analysis applied either in a sliding-window or in a cumulative mode. Furthermore, we demonstrate the possibility of monitoring transient or unexpected neural activities and suggest that real-time ICA may provide the fMRI researcher with a better understanding and control of subjects' behaviors and performances.
\end{abstract}

(C) 2003 Elsevier Inc. All rights reserved.

Keywords: Functional magnetic resonance imaging; fMRI; Real-time analysis; Exploratory data-driven analysis; Descriptive statistics; Sliding-window analysis; Independent component analysis; Fixed-point algorithm; Receiver operating characteristics

\section{Introduction}

Real-time functional magnetic resonance imaging (fMRI) is a promising tool for the noninvasive monitoring of brain activity during an ongoing imaging session. In the

\footnotetext{
* Corresponding author. Department of Neurological Sciences, University of Naples Federico II, II Policlinico (Nuovo Policlinico) Padiglione 17, Via S. Pansini 5, 80131 Naples, Italy. Fax: +39-081-546-3663.

E-mail address: faesposi@unina.it (F. Esposito).
}

recent past, various efforts have been made to develop favorable acquisition strategies (Yoo et al., 1999) and to reformulate conventional off-line analysis techniques (Cox et al., 1995; Gembris et al., 2000; Posse et al., 2001) to permit the highly computationally demanding real-time applications.

In fact, one major issue of real-time methodology is to find an optimized trade-off between the accuracy in estimating neural activity and the ability of performing the estimation within computational times compatible with the 
temporal rate at which fMRI time-series are acquired. Conventional fMRI data analysis methods impose the collection of a minimum batch of temporal observations in order to generate a reliable activation map. For a given repetition time $(T R)$, the longer is the time window covered by the input data set, the more data points will be collected that will improve the statistical identification of activation phenomena. On the other hand, extending the window of data collection, the ability of conventional data analysis methods to detect transient or temporally nonstationary dynamic effects in the time-series will be strongly sacrificed in favor of repetitive and temporally stationary effects (Mitra and Pesaran, 1999).

Furthermore, the computational load of the current methods increases with the number of time points to be processed, thus limiting feasibility and benefits of the real-time processing setting. The main challenge for candidate realtime analysis techniques is that the calculations are to be completed within a specified fixed and short time (Cox et al., 1995). This practically requires the ability to process fMRI time-series within times comparable to those required for ordinary image acquisition, reconstruction, and network transmission. As the most demanding case, here we assume that a real-time fMRI analysis needs to be able to generate task-related activation maps within time spans in the order of one TR of the acquisition sequence. So far, only standard univariate statistics like correlation (Bandettini et al., 1993) and multiple regression analyses (Friston et al., 1995a) have been successfully employed for real-time analysis of fMRI data. They utilized two different approaches: cumulative (Cox et al., 1995) and sliding window (Gembris et al., 2000; Posse et al., 2001).

In the cumulative approach, the correlation coefficients between the partial time-series of a reference vector representing the expected hemodynamic response and the measured time-series in each voxel are computed in a cumulative manner, whereas the same vectors are growing in length with each newly acquired volume. In this approach, one edge of the window of collection is fixed, whereas the other moves during the acquisition of new data. The specificity of this approach typically increases over time because the number of false positives becomes smaller as more data become available for averaging. On the other hand, the sensitivity of the approach will be reduced, if, across repeated trials, all the responses are regarded as a sole statistical ensemble and if significant trial-by-trial fluctuations are neglected (Mitra and Pesaran, 1999).

In the sliding-window approach, the computation of correlation statistics is restricted to the most recently acquired functional data. This means that both edges of the window of collection move during the acquisition. The accuracy of this approach is, thus, constant over time and dynamic changes in brain activity can be resolved in dynamically varying activation patterns. On the other hand, due to the limited signal-to-noise ratios, the overall specificity will be strongly affected by the reduced observation and collection times.

For both the approaches the accuracy can be improved by using a real-time motion correction technique (Mathiak and Posse, 2000) and an optimized modeling of the reference and trend signals (Gembris et al., 2000; Posse et al., 2001). Further developments in real-time analysis and representation of fMRI data may comply better with the complexity of neural responses. This may extend far beyond the strict predictability of task-related activities by using more flexible processing methods.

The key limits of univariate hypothesis-driven methods is that they rely solely on the temporal predictability of the phenomenon to be detected, neglecting the information deriving from the covariance of the acquired voxels' timeseries, even if univariate approaches may be formulated independent of a temporal model.

On the contrary, multivariate analyses (Friston et al., $1995 b)$ depend strongly on the voxels' covariance. These methods, and in particular the Independent Component Analysis (ICA) used in this article, are, in principle, more flexible than univariate ones. In fact they provide extensive information about a number of possible signals existing in an image time-series, including those that would be difficult to formalize by means of an a priori temporal model, even if multivariate methods can also be formulated based on temporal models. In fact, the multivariate approaches allow to characterize those neural phenomena that generate a nonzero mutual correlation among voxel time courses from different interconnected areas and are often combined with descriptive data-driven techniques (Sychra et al., 1994; Friston, 1995b; Bullmore et al., 1996) to provide more complex and insightful representations of the data.

At present, multivariate and descriptive analysis techniques have not been applied to real-time fMRI. Two major drawbacks of these techniques that practically limit their use within a real-time analysis framework are the computational load (that is considerably higher than that of univariate analysis techniques) and the difficulties in selecting and interpreting the results arising from the large number of different phenomena detected. Independent Component Analysis (ICA) is one of the most promising approaches to the off-line multivariate and data-driven analysis of fMRI data (McKeown et al., 1998a; Brown et al., 2001). ICA decomposes blindly the acquired voxels' time-series into a set of spatial maps and associated time courses. In its spatial variant (McKeown et al., 1998a, 1998b; Calhoun et al., 2001), it exhibits the fundamental property of finding datadriven representations of functional measurements relying mainly on the spatial features of neural activities rather than the temporal features of the recorded signals.

As for principal component analysis (PCA) (Friston et al., 1993; Andersen et al., 1999), another multivariate analysis technique, the first step of the analysis typically requires the computation of the standard covariance matrix of the voxels' time courses included in the analysis. The ei- 
genstructure of the covariance matrix is, then, used to generate the orthogonal directions along which the input timecourses are projected, resulting in the output maps. The information content of the covariance matrix refers strictly to the temporal window of observation; thus the method relies on the number of collected time points. Nevertheless, spatial ICA only models the spatial distributions of brain activities (Everitt and Bullmore, 1999; Esposito et al., 2002) and builds accordingly the temporal filters that produce the statistically independent maps (the independent components). Hence, even two time points of the volume of interest may be sufficient to start the ICA algorithm and possibly yield a reliable descriptive representation of the factors that cause the measured signal changes (commonly referred to as "sources"). This suggests that, in contrast to conventional approaches that improve in accuracy with time only if more observations are available, spatial ICA bears the potential of providing meaningful results even if starting from highly reduced data sets, allowing a fast and automatic dynamic analysis of fMRI time-series, possibly during the same imaging session.

Furthermore, the computational load of a spatial ICA algorithm's iteration grows much more with the temporal dimension than with the number of voxels included in the analysis (Brown et al., 2001). This makes it rationale to implement real-time ICA in a sliding-window approach. Although the number of active brain sources does not depend conceptually on the number of time points, this number conditions the maximum number of components that can fit a complete ICA model. As a consequence, working on short (moving) windows has the disadvantage of reducing correspondingly the number of sources that can be represented but makes the display, the selection, and the interpretation of the components easier and faster, which is necessary in a real-time setting.

Recently, a fundamental connection existing between ICA as a general theory and Exploratory Projection Pursuit (EPP) (Friedman, 1986) has been pointed out in the context of fMRI data analysis (Suzuki et al., 2002). In this study, a direct search method, estimating the components one by one with simple and tailored contrast functions, has been shown to improve dramatically the computational efficiency of the method, facilitating the off-line extraction of desired or interesting components.

Here we applied spatial ICA and the direct search method as a real-time processing tool for fMRI data analysis in a sliding-window approach. We demonstrate that it is possible with these methods to achieve dramatic reductions in the time required to extract ICA components. We show that at each time point, a one-by-one search of meaningful spatially independent projections of short time courses can be computed in tens or hundreds of milliseconds per projection and per slice. Moreover, cumulative maps and time courses are easily constructed by serially averaging in realtime selected patterns (see Materials and methods for details about the selection process), which result from consecutive runs of the sliding-window decompositions. In practice, although the dynamically generated components are used to monitor the occurrence of both expected and unexpected functional events, the cumulative readouts keep track of the temporal evolution of those activation phenomena that turn out to be stationary across repeated trials (Fig. 1). Our proposed technique is tested and compared with classical regression analysis on real and simulated data. a thresholdindependent methodology (receiver operator characteristics, ROC (Skudlarski et al., 1999; Esposito et al., 2002)) has been used to quantify the performances.

\section{Materials and methods}

\section{The ICA data model and the Direct Search algorithm}

The basic definition of the spatial ICA model is that $P$ time-course vectors (each corresponding to one of $P$ selected voxels in a reference image) in a $T$-dimensional space of time courses ( $T$ being the number of time points included in a temporal window of collection) are linearly mapped to $P$ vectors in a $K$-dimensional space (i.e., the space of the Independent Components or ICs), $K$ being less or equal than $T$ as follows:

$$
\mathbf{x}(p)=\mathbf{A} \cdot \mathbf{s}(p) \quad p=1, \ldots, P
$$

where, the " $\mathrm{T}$ " denotes the transpose, $\mathbf{x}(p)=\left[x_{1}(p), \ldots\right.$, $\left.x_{\mathrm{T}}(p)\right]^{\mathrm{T}}$ is the observed time-course for voxel $p$ and $\mathbf{s}(p)=$ $\left[s_{1}(p), \ldots, s_{\mathrm{K}}(p)\right]^{\mathrm{T}}$ is a $K$-fold set of statistically independent variables, observed at each voxel $p$, defining the spatially independent maps (ICs). By definition (Papoulis, 1991), this means that:

$$
f\left(s_{1}, s_{2}, \ldots, s_{K}\right)=\prod_{i=1}^{K} f_{i}\left(s_{i}\right)
$$

$f$ and $f_{i}$ being, respectively, the joint probability density function of $\mathbf{s}$ and the marginal probability density function of the generic component $s_{\mathrm{i}}$. The $T \times K$ unknown matrix $\mathbf{A}$ in Eq. (1), called the mixing matrix, is assumed to be invertible, and each of its columns, which correspond to a basis vector of the new space of the ICs, represents a time course of activation (TC).

A generic ICA algorithm addresses the problem of assessing the model (1) by seeking for an unmixing $K \times T$ matrix $\mathbf{W}$ so that the following vector:

$$
\mathbf{y}(p)=\mathbf{W} \cdot \mathbf{x}(p)
$$

is an estimate of the hidden variables $\mathbf{s}(p)$, except for permutations, signs, and amplitudes. Matrix $\mathbf{A}$ can be computed as the pseudoinverse of $\mathbf{W}$.

The ICA model estimation problem has been originally approached by measuring the amount of statistical dependence within a fixed number of estimated components and minimizing it using an iterative (adaptive at a more or less 


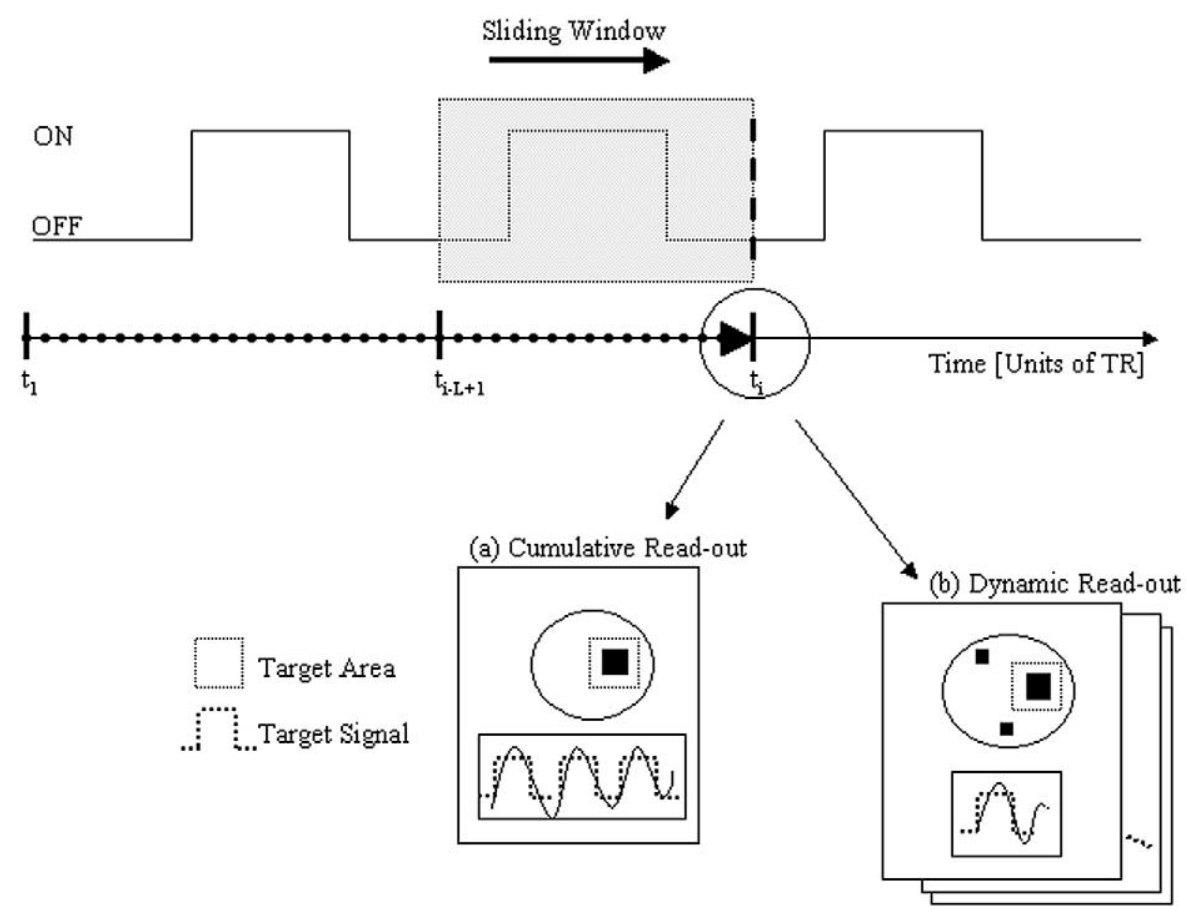

Fig. 1. Basic diagram for real-time independent component analysis of functional MRI time-series. The gray shading indicates the width of the sliding window. At the generic time point $t_{i}$, only the frames sampled in this temporal interval are involved in real-time ICA calculations and readout updates. (a) The cumulative map and time-course represent phenomena that pertain the measured time-series from $t_{l}$ to $t_{i}$. (b) The sliding-window maps and time courses represent phenomena that pertain the measured time-series from $t_{i-L+1}$ to $t_{i}$ and do not cumulate over time.

degree) learning algorithm. Mutual information (MI) is the fundamental theoretical function that has been introduced by Comon (1994). In the first application of ICA to fMRI time-series (McKeown et al., 1998a) the infomax approach was used and the minimization of mutual information was achieved according to the infomax principle (Bell and Sejnowski, 1995). The fixed-point algorithm (Hyvärinen, 1999) pursues the same goal using the concept of normalized differential entropy or negentropy, an earlier theoretical function (Comon, 1994), usefully interpreted as a measure of non-Gaussianity of a distribution.

We reviewed and compared the use in fMRI data analysis of the infomax algorithm and a correspondent symmetric version of the fixed-point algorithm in Esposito et al. (2002). Here we exploit the fact that the fixed-point algorithm can be used to estimate not only a fixed number of components in parallel (symmetric approach) but also a varying number of components one by one (hierarchical approach). The two different version of the fixed-point algorithm are respectively obtained by employing the $\mathrm{Ne}$ gentropy as multiunit or a one-unit objective function (Hyvärinen, 1999).

In the hierarchical approach, each single row of $\mathbf{W}$, say $\mathbf{w}^{\mathbf{T}}$, is estimated one at a time in a way of exploratory projection pursuit (EPP, (Friedman, 1986)). Thus, a single scalar independent component (IC) is easily obtained as follows:

$$
y(p)=\mathbf{w}^{\mathbf{T}} \cdot \mathbf{x}(p)
$$

In the fixed-point ICA algorithm (Hyvärinen, 1999), Maximum Entropy Principle-(MEP) based approximations of negentropy (Hyvärinen, 1998) lead to the following general form of a one-unit contrast function $J_{G}$ as follows:

$$
J_{G}(\mathbf{w})=\left[E\left\{G\left(\mathbf{w}^{\mathbf{T}} \mathbf{x}\right)\right\}-E\{G(v)\}\right]^{2},
$$

where $E\{\cdot\}$ is the expectation operator, $\mathbf{w}^{\mathbf{T}}=\left[w_{1}, \ldots\right.$, $\left.w_{\mathrm{T}}\right]^{\mathrm{T}}$ is a weight vector (a row of the matrix $\mathbf{W}$ ) under the constraint $E\left\{\left(\mathbf{w}^{\mathbf{T}} \mathbf{x}\right)^{2}\right\}=1, G$ is nonquadratic function (see below), and $v$ is a zero-mean Gaussian variable with unit standard deviation. The problem of finding a single IC is, thus, solved by finding a local maximum of the form in Eq. (5), after sphering the data by means of a standard principal component analysis (PCA) as follows:

$$
\hat{\mathbf{x}}(p)=\mathbf{B} \cdot \mathbf{x}(p) .
$$

The $T \times T$ matrix $\mathbf{B}$ in Eq. (6) is called the sphering matrix and is easily determined through the following formula:

$$
\mathbf{B}=\mathbf{D}^{-1} \cdot \mathbf{E},
$$

where $\mathbf{E}$ is the $T \times T$ transposed matrix of the eigenvectors and $\mathbf{D}$ the diagonal matrix of the eigenvalues of the covariance matrix of the input data $E\left\{\mathbf{x} \mathbf{x}^{\mathrm{T}}\right\}$.

When using a one-to-one hierarchical estimation approach, the order in which the single projections are estimated is highly important in a real-time fMRI application because the first components to be extracted are also the first 
results that become available. Influencing this order can be done optimally by choosing function $G$ and posing favorable initial conditions, because any optimization algorithm tends to first find the local maxima of the objective function that have largest basins of attraction and that are closest to the initial conditions.

This approach has been recently reviewed (Suzuki et al., 2002) and has empirically driven the final choices for function $G$, leading to fast and precise off-line estimations of fMRI activation components (Suzuki et al., 2002).

The main properties of function $G$ are asymmetry and sparsity from one side and the computational simplicity from another side. A sufficiently general form of these functions, proposed and discussed in Hyvarinen (1999), is as follows:

$$
G_{n}(u)=\frac{u^{n}}{n} \quad n=3,5, \ldots, \text { any odd integer. }
$$

The case $n=3$ leads to a maximization of the third-order cumulant or skewness of the target IC and is a fundamental measure of the asymmetry of a statistical distribution. Higher order odd functions preserve the property of asymmetry and are more sensitive to the tail of the distributions (sparsity) even if they lead to a poorer approximation of the original negentropy. $G_{1}$ and $G_{5}$ have been used in our implementation as in Suzuki et al. (2002).

The maximization of $J_{G}$ in Eq. (5) with the Newton's optimization method gives us the basic iteration steps of the fixed-point algorithm in deflation mode (Hyvarinen, 1999) as follows:

$$
\begin{aligned}
& \hat{\mathbf{w}}^{+}=E\left\{\hat{\mathbf{x}} \cdot G^{\prime}\left(\hat{\mathbf{w}}^{\mathrm{T}} \hat{\mathbf{x}}\right)\right\}-E\left\{G^{\prime \prime}\left(\hat{\mathbf{w}}^{\mathrm{T}} \hat{\mathbf{x}}\right)\right\} \hat{\mathbf{w}} \\
& \hat{\mathbf{w}}^{*}=\hat{\mathbf{w}}^{+} /\left\|\hat{\mathbf{w}}^{+}\right\|,
\end{aligned}
$$

where $\|\cdot\|$ denotes the euclidean vector norm and $\mathbf{w}^{*}$ is the updated value of $\mathbf{w}$, the basis vector of the corresponding independent component estimate $y$ in Eq. (4) (Hyvarinen, 1999; Suzuki, 2002). For what concerns the initial conditions, we either used random entries for the vector $\mathbf{w}$ or fully followed the direct search procedure (Suzuki et al., 1999) in that the vector $\mathbf{w}$ was to be first initialized according to the following:

$$
\mathbf{w}=\mathbf{B} \cdot \mathbf{b},
$$

where $\mathbf{b}$ is a column T-vector representing a target zeromean (see below) signal change in the window.

Equation (9) is repeated until the mean square change in vector $\mathbf{w}$ becomes less than a specified tolerance $\varepsilon$ (e.g., $\varepsilon<$ $10^{-6}$ ), within a maximum total number of iterations (e.g., 100). Once convergence for one component is achieved, the next component is searched after back-projecting and serially removing the last estimate from the whitened data (Suzuki et al., 2002) as follows:

$$
\hat{\mathbf{x}}_{\text {new }}(p) \leftarrow \hat{\mathbf{x}}(p)-\mathbf{w}_{1} \cdot \mathbf{w}_{1}^{\mathbf{T}} \cdot \hat{\mathbf{x}}(p),
$$

where $\mathbf{w}_{1}$ is the last estimated IC basis vector and the subscript new indicates the data for the subsequent estimate.

\section{Real-time ICA of fMRI time-series: dynamic and cumulative effects}

The real-time application of the ICA framework requires the setting of the three independent parameters that define the input space of the data vectors. These settings pertain the temporal interval of data collection (i.e., the length of the window), the repetition time of the scans involved in the calculations (that corresponds to a decimation factor of the time-series), and the desired step of the update process. All these temporal parameters are to be expressed in units of time or TR (i.e., time points of acquisition). Let us denote by $L$ (length of temporal window), $D$ (decimation factor), and $S$ (step for the scenario update) these parameters.

The basic processing scheme operates in a way that, once the window is filled up with new data, the one-unit fixedpoint algorithm is launched. The temporal dimension $T$ of our dynamic analysis equals the actual number of scans included in the window as follows:

$$
T=\text { integer }\{L / D\}
$$

and, after $S$ newly acquired time points, a varying number of IC maps are generated, ranging from 0 (in case no convergence is achieved within the maximum number of iterations) to a maximum number of $\mathrm{T}$ independent components. To simplify we assume here that $D=S=1$ and so $L=T$; the extension to more general cases is straightforward. At time point $i$, the voxel observation is, then, represented by the $L$ most recent time points as follows:

$$
\mathbf{x}^{(i)}(p)=\left[\begin{array}{c}
x_{i-L+1}(p) \\
\cdots \\
x_{i}(p)
\end{array}\right] .
$$

For each basis vector serially estimated by the algorithm in Eq. (9), the corresponding spatial map can be immediately displayed and represents one of a maximum of $L$ detected activation phenomena (see Results and Discussion). These spatial maps are referred to as dynamic maps, because they reflect phenomena occurring during only the most few recent time points at the current position of the window and cease to be representative when the new time point arrives.

In order to generate the dynamic map, the IC $y(p)$ is determined following Eq. (4), spatially normalized to unit standard deviation and eventually thresholded and colorcoded. Before starting the entire process (acquisition and calculations), and depending on the experimental design, one or more spatial ICs can be targeted by using appropriate initial entries for vector $\mathbf{w}$ in the initialization step [imposing a temporal "a priori" through vector $\mathbf{b}$ in Eq. (10)] or specifying an anatomical region of interest (ROI) within the volume of acquisition (i.e. a spatial "a priori", see below). 
The most natural choice for initial conditions may be derived from a simple and classical reference vector $\mathbf{r}=$ $\left[r_{1}, \ldots, r_{i}, \ldots\right]$, restricted at each run to the current window of collection. This may correspond to serially updating vector $\mathbf{b}$ in Eq. (10) directly from task performance or behavioral measures in parallel to the acquisition (Voyvodic, 1999). Optionally, and depending on the temporal setting, this vector incorporates typical hemodynamic shaping and delays.

The temporal "a priori" clearly biases the search process toward the ICs that mostly match the temporal change expressed by the tentative reference vector and enable a rapid estimation of those components that are expectable or predictable at a certain degree. On the other hand, this bias cancels out if unpredictable events, different from the phenomenon that was temporally coded in vector $\mathbf{b}$, strongly affects the data structure in a way that heavily attracts the point of convergence of the one-unit algorithm. Alternatively, vector $\mathbf{b}$ can be initialized with just random entries; this choice removes the temporal bias and none of the signal sources are favored.

Targeting one IC opens the possibility for tracking the cumulative history of the underlying activation phenomenon. This is done practically by producing increasingly accurate spatial maps over the entire time of the real-time fMRI session. Suppose that, at the $i$ th time point of work, we have extracted, selected (see below), and stored a timeseries of ICs and associated TCs that are defined throughout the series of $L$ scans of the temporal window. We can denote by $k_{i}$ the index of the selected component (intended as IC and TC) as follows:

$$
\begin{gathered}
y_{k_{1}}^{(1)}(p), \ldots, y_{k_{i-2}}^{(i-2)}(p), y_{k_{i-1}}^{(i-1)}(p), y_{k_{i}}^{i}(p) \\
{\left[\begin{array}{c}
a_{L}^{(1)} \\
\cdots \\
a_{1}^{(1)}
\end{array}\right] \cdots,\left[\begin{array}{c}
a_{L}^{(i-2)} \\
\ldots \\
a_{1}^{(i-2)}
\end{array}\right],\left[\begin{array}{c}
a_{L}^{(i-1)} \\
\ldots \\
a_{1}^{(i-1)}
\end{array}\right],\left[\begin{array}{c}
a_{L}^{(i)} \\
\cdots \\
a_{1}^{(i)}
\end{array}\right] .}
\end{gathered}
$$

In general, for a selected component, the uncertainty in the sign of the maps and associated time courses can be easily solved by back-projecting each estimated IC with the corresponding TC down to the underlying measured voxels (Duann et al., 2002) and simply inverting the signs of the ICs and TCs when the correlation coefficient of the positively active voxels (see below) and the TCs are negative. Thereafter, a cumulative map is constructed by serially averaging all the collected ICs as follows:

$$
y_{i}^{\text {cum }}(p)=\frac{1}{i} \sum_{j=1}^{i} y_{k j}^{(j)}(p)
$$

and a cumulative time course is constructed through a timeby-time average of the estimated TCs as follows:

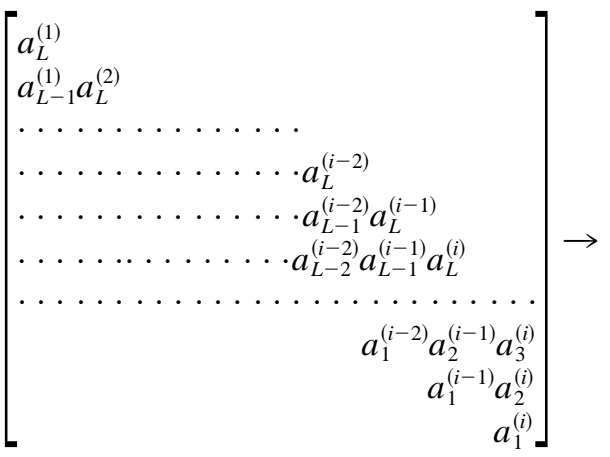

$$
\begin{aligned}
& \begin{array}{c}
{\left[\begin{array}{l}
a_{L}^{(i)} \\
\left(a_{L-1}^{(1)}+a_{L}^{(2)}\right) / 2 \ldots \ldots \ldots \ldots \ldots \ldots \\
\cdots \ldots \ldots \ldots \ldots \\
\left(a_{1}^{(i-L-1)}+\ldots \ldots+a_{L}^{(i-2)}\right) / L \\
\left(a_{1}^{(i-L)}+\ldots+a_{L-1}^{(i-2)}+a_{L}^{(i-1)}\right) / L \\
\left(a_{1}^{(i-L+1)}+\ldots+a_{L-2}^{(i-2)}+a_{L-1}^{(i-1)}+a_{L}^{(i)}\right) / L \\
\cdots \ldots \ldots \ldots \ldots \\
\left(a_{1}^{(i-2)}+a_{2}^{(i-1)}+a_{3}^{(i)}\right) / 3 \ldots \ldots \ldots \\
\left(a_{1}^{(i-1)}+a_{2}^{(i)}\right) / 2 \\
a_{1}^{(i)}
\end{array}\right]} \\
=\left[\begin{array}{l}
a_{1}^{\text {cum }} \\
a_{2}^{\text {cum }} \\
\cdots \\
a_{i-L-1}^{\text {cum }} \\
a_{i-L}^{\text {cum }} \\
a_{i-L+1}^{\text {cum }} \\
\cdots \\
a_{i-2}^{\text {cum }} \\
a_{i-1}^{\text {cum }} \\
a_{i}^{\text {cum }}
\end{array}\right] .
\end{array}
\end{aligned}
$$

This forms preserves the number of estimates that have been so far performed through the sliding-window mechanism and gives back a cumulative read-out of the ongoing brain activity.

\section{Real-time ICA of fMRI time-series: display and representation issues}

Two preliminary choices are needed by the real-time ICA application: (1) the maximum number of ICs that are to be extracted and displayed as dynamic maps at each run [the default case in our performance analysis was $K=T=L(D$ $=1)$ ] and (2) the number of ICs that are to be used to generate maps and time-courses in the cumulative output.

The first choice relates to the existing trade-off between the effective computational load (directly dependent on the window parameters $L, D$, and $S$ and the acquisition TR) and the number of distinct brain activities that is desired to follow in the real-time update of the output scenario. The second choice is substantially driven by the experimental design. The final outcome of the accumulation will be strictly related to the success of the dynamic selection process. Reordering and selecting the components starting from 
the natural order of extraction is a general problem in the ICA of fMRI data (Gu et al., 2001; Formisano et al., 2002) and becomes crucial for the real-time extension of ICA.

The simplest way to select an IC would be to assess the temporal linear correlation between each IC's representative time course and the windowed reference vector. This is commonly done in off-line ICA as a trivial selection step. Unfortunately, also in the presence of an adequate temporal model of signal changes, this approach will not generally provide a reliable criterion to select the target components, because the use of short temporal windows (or, in general, the use of few scans within a given temporal window), although speeding the decomposition algorithm and improving the real-time feasibility, will also dramatically decrease the power of this method. Moreover, it cannot be used when the protocol is not easily coded in a reference vector or even not known. On the other hand, various ranking criteria have been proposed so far in the literature (Gu et al. 2001; Formisano et al., 2002). These criteria, which have been shown to be effective for ranking and selection of ICA off-line estimates, generally rely on the spatiotemporal structure of ICs and no temporal models of the signal changes need to be applied to the basis vectors.

Although the cited selection strategies could be easily implemented at variable computational costs, we finally adopted a region of interest-(ROI) based selection criterion for the real-time ICA. A region of interest is roughly specified by the user after a set of anatomical reference scans or few training functional scans of the same experiment. During a preliminary session, one or more areas are localized anatomically or functionally using standard methods (Castelo-Branco et al., 2002) and labeled as areas of primary interest. Thereafter, when the real experimental session starts and the real-time ICA is launched, each IC generated by the direct search process is assigned with a score according to the numbers of active voxels that are adjacent to each other in the selected ROIs. This score was used here for the selection of the IC and the consequent update of the cumulative maps. In any case it could also act as an additional criterion for stopping the runs before the maximum number of allowed ICs is reached.

\section{Image acquisition and simulation}

Three right-handed healthy volunteers (AA, FDS, FE; ages 25-40 years) participated in three sessions of a dominant-hand finger-tapping fMRI experiment. Images were acquired on a 1.5-T super-conducting SIGNA MR scanner (General Electric Medical Systems, Milwaukee, WI, USA) using a standard circularly polarized head coil. $T_{1}$-weighted structural volumes served as anatomical reference in order to position four slices parallel to the bicommissural plane and to cover optimally the primary motor and supplementary motor areas. The functional scans were acquired using a conventional gradient-echo echo-planar imaging sequence (TR, $2 \mathrm{~s}$; echo time $T E, 60 \mathrm{~ms}$; delay time, $2 \mathrm{~s}$; flip angle $90^{\circ}$, field of view $210 \mathrm{~mm}$, matrix $128 \times 128$, slice thickness $5 \mathrm{~mm}$, slice gap $2 \mathrm{~mm}$ ). The experimental paradigm consisted of 10 blocks of five volumes during which a selfpaced finger-tapping task (sequential opposition of all fingers of the right hand against the thumb) at a specified frequency of $2 \mathrm{~Hz}$ was carried out and 10 blocks of five volumes during resting. The alternation between task and rest conditions was verbally triggered and the frequency and quality of the task controlled by visual inspection.

Artificial fMRI time-series were created by adding activation patterns to a separate data set of 100 echo-planar slices collected in one of the subjects during a constant rest (null) condition. The spatial layouts of the simulated activation were obtained from the spatial layouts of the regions found to be activated by the motor task (primary motor cortex and supplementary motor area) and detected by conventional linear correlation analysis (Fadili et al., 2000). At each selected voxel within these regions, a simulated activation time course was injected in the null data set using an additive model. Those signals have been parameterized in terms of the maximum signal enhancement, $\Delta S$, divided by the average image intensity $S$ (activation contrast level, $\mathrm{ACL}=\Delta S / S$ (Gu et al., 2001)), which typically ranges from 0.5 to $2 \%$, at $1.5 \mathrm{~T}$ at a conventional voxel size (Bandettini et al., 1992; Kwong et al., 1995).

Four simulated data sets were, thus, generated, each having a different value for the activation contrast level $(\mathrm{ACL}=0.5,1,1.5$, and $2 \%)$. The simulated fMRI responses consisted of a boxcar-shaped waveform convolved with a gamma kernel having parameters $\delta=2.5 \mathrm{~s}$ and $\tau=$ $1.5 \mathrm{~s}$ (Boynton et al., 1996). In addition, to simulate respectively the typical spatial variations of the hemodynamic response and the physiologic fluctuation of task performance, a stochastic delay (mean 0 and standard deviation $2.5 \mathrm{~s})$ was added to the gamma function prior to the convolution and a white gaussian noise (SD $=2 \%$ of estimated baseline noise) was added to the signal prior to adding the activation to a voxel (Gu et al., 2001).

\section{Data analysis and accuracy evaluation}

All experimental data were processed in a Matlab environment. A graphic user interface has been developed to make selecting the network folders, specifying the running slice and the ROIs, setting appropriate protocols and preand postprocessing parameters, and choosing ICA run and display easier. Although the implemented program allows multislice acquisition and processing, single-slice ICA runs have been performed and compared to single-slice off-line ICA runs (see below). Nevertheless, the running slice can be dynamically changed during a run. Alternatively, the decomposition of a multislice time-series can be performed: this increases the number of voxels that undergo the analysis and has the potential to improve the decomposition at the cost of a linear increase of the computational time. 
Before entering the core statistical processing routines, each image of the running slice time course was optionally smoothed in space with bidimensional Gaussian kernels. Then the voxels outside the brain were excluded by using a simple intensity threshold masking procedure, whereas the remaining voxels were used to fill the data matrix whose columns corresponded to the observation vectors $\mathbf{x}$ in Eq. (1). Such a matrix was finally given as input to the ICA routine function, whose Matlab implementation has been adapted from the basic source code downloaded from the Internet (http://www.cis.hut.fi/projects/ica/fastica/) according to the sliding-window direct search approach described in the previous paragraph.

We repeated our tests for two different contrast functions $\left(G_{3}\right.$ and $\left.G_{5}\right)$. For each step, the maximum number of iterations was set to 100 and a minimum root-mean-square change of 0.0001 was allowed for the convergence of IC estimates. The window length was set to 10 scans (i.e., an interval that equals the period of the stimulation). No decimation of the time courses was performed $(D=1)$. With these indicative settings, the maximum elapsed times to cover the 100 iterations for the extraction of the 10th independent component of a one slice time course was always limited to $4 \mathrm{~s}$ on a computer running Windows 2000 (Pentium III $750 \mathrm{MHz}, 512 \mathrm{MB}$ RAM). As soon as the ICs set was produced, the cumulative map and the cumulative time course were automatically updated. If no independent components could be extracted within the specified iterations, all the displayed patterns remained unchanged in the scenario.

Sliding-window and cumulative linear regression analysis have been performed on the same data for comparison and validation purposes. The underlying linear models optionally included a first-order (linear) detrending (Cox et al., 1995; Posse et al., 2001) within a general linear model analysis (GLM; Friston, 1995a). As for the ICA analysis, no motion-correction techniques were implemented. At each time point, after each run, the natural ranking of the ICs of interest as well as the elapsed times were recorded.

An ROC analysis (Skudlarski et al., 1999) of the results on both simulated and real activation data sets was performed for all the dynamic and cumulative maps as follows. For each selected ICA map, a ROC curve was first determined by combining the false-positive fractions (FPFs) and the false-negative fractions (FNFs) at a varying threshold. Then, after curve fitting, the ROC power (i.e., the mean of the ROC curve over he range of FPFs from 0 to 0.01 ) has been utilized as a synthetic threshold-independent figure of merit of the spatial accuracy. The resulting values were, averaged across time for the dynamic maps and assembled in a ROC time-course for the cumulative maps. For the real activation time-series, the "off-line" activation maps $(P<$ 0.01 ), produced respectively by the linear regression analysis and the independent component analysis, were used for the definition of true positives and true negatives.

For the purpose of the off-line ICA, the infomax algo- rithm has been used since both infomax and fixed-point algorithms produce similar and accurate results in their "off-line" application to fMRI data (Esposito et al., 2002).

On the contrary, the use of the infomax algorithm for the real-time ICA is less convenient since it requires to specify in advance the number of components to be extracted and would not give any output in case not all these components were estimated within the interval between two successive scans.

In order to assess the impact of the initialization on the measured performances of real-time ICA, multiple runs ( $n$ $=10$ ) of the "off-line" ICA on one representative data set with randomized initial conditions were performed. This yielded a mean standard deviation of about $1 \%(0.011 \pm$ 0.003 ) among the time points of the ROC power time-series.

Rectangular ROIs of 300-400 pixels were specified on the reference scan for each experimental data set that included the target areas as identified by the off-line methods.

\section{Results and discussion}

\section{Computational times and general feasibility of real-time ICA}

Fig. 2 shows a comparison of computational times between the sliding-window and a cumulative application of ICA on a simulated data set $(\mathrm{ACL}=2 \%)$. Although the number of iterations required for the extraction of a target IC remains practically constant (Fig. 2a), the cumulative ICA imposes elapsed times that, at a rough estimate, grow linearly with the number of scans (Fig. 2b). On the contrary, those times are short and constant for the sliding window ICA (Fig. 2b). Nonetheless, ROC measures on the cumulative maps generated by progressively averaging the output of the sliding-window ICA according to Eq. (15) are comparable and convergent with ROC measures performed on purely cumulative ICA maps (Fig. 2c). Elapsed times (mean and standard deviations over 91 scans) for the extraction of the target IC (here selected as the activity that is also extracted by a conventional inferential approach) are reported in Fig. 3.

It is important to note that further relevant reductions of the elapsed times are observable by applying a decimation factor $(D>1)$ to the temporal dimension of the analysis. Although we generally dimensioned the sliding window on the length of the blocks of activation and resting, the number of time points per window can be reduced to a subset of the window length, yielding a further relevant reduction of calculation time without affecting the quality of the decomposition. Here the real-time ICA approach has been implemented using Matlab routines and proved to be compatible with a real-time application, the elapsed times being in the order of hundreds of milliseconds per component.

Quantitative results for the accuracy of real-time ICA are reported in Figs. 4, 5, and 6 and discussed below. The ROC 


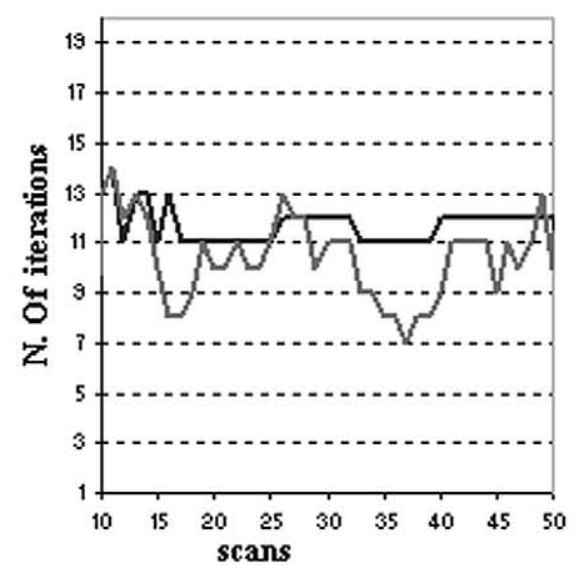

(a)

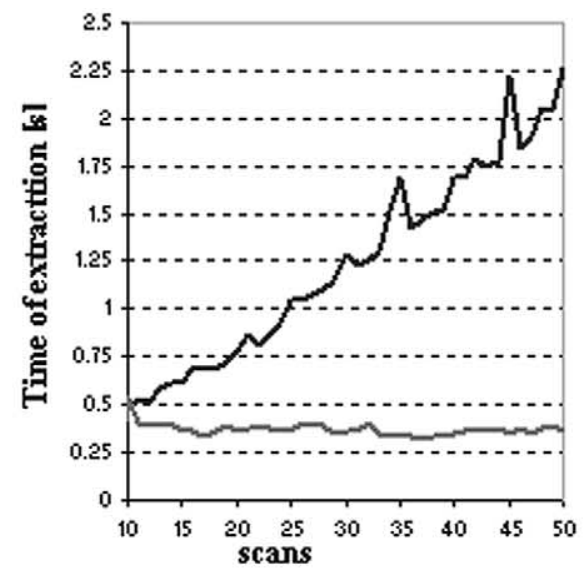

(b)

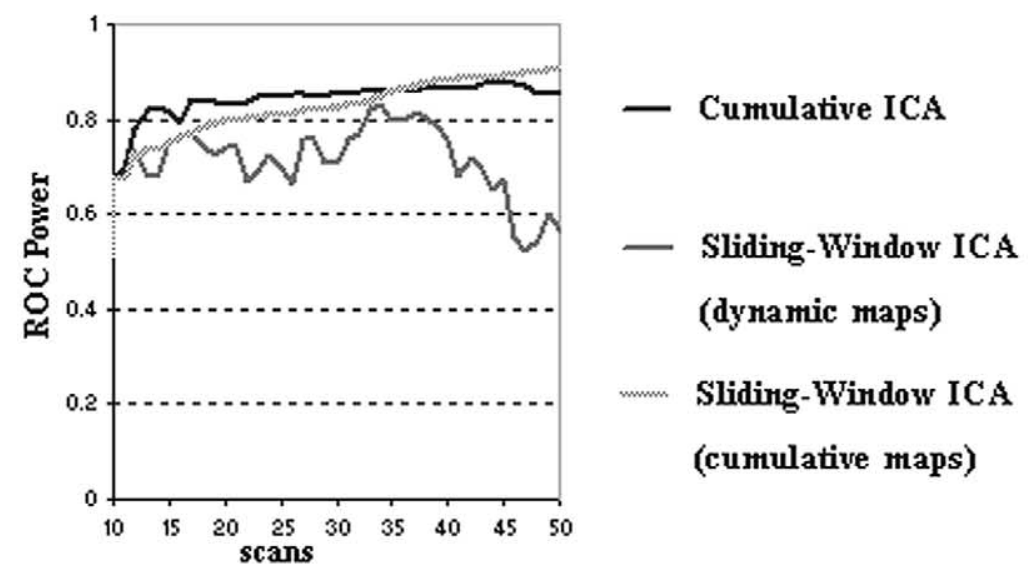

(c)

Fig. 2. Comparison between sliding-window ICA and cumulative ICA on a representative data set. (a) The number of iterations required to extract the target IC Is reported as a function of the scan for the two alternative approaches to update the data matrix. (b) Corresponding times of extraction of the target IC. (c) ROC-measured accuracy for the maps generated from a cumulative approach and for the dynamic and cumulative maps generated from the sliding-window approach.

power was computed for two different contrast function $\left(G_{3}\right.$ and $G_{5}$ ) used in the ICA decompositions and with and without linear trend signals adopted in the GLM analyses. The accuracy of the real-time methods has been separately assessed for different activation contrast levels and different subjects. The capability of the proposed method in dynamically detecting task-related changes and tracking a cumulative spatiotemporal pattern of activation from a blockdesigned motor experiments is quite evident.

A general observation concerns the optional use of a simple bidimensional Gaussian smoothing filter ( $\mathrm{FWHM}=3$ pixels) to the images: its use on our experimental data always improved the ROC power of the activation maps while not affecting significantly the processing time (Fig. 3). On the other hand, a spatial smoothing could also blur and filter out transient and and subtle spatial activations that a real-time analysis can detect better than an off-line analysis.

In our setting for the real-time ICA, we generically considered as "failures" all the frames where no accurate task-related (see below) dynamic maps were successfully selected within the the interval between two successive scans. The method demonstrated a good reliability $(>90 \%)$ at $\mathrm{ACI} \geq 1 \%$, because the failures ranged from 8 of 91 scans (reliability of $91.2 \%$ ) at ACL $=1 \%$ to 2 of 91 scans (reliability of $97.8 \%$ ) at ACL $=2 \%$ in our tests. Notably, the corresponding cumulative patterns were not significantly affected by these failures.

\section{Performances of real-time ICA on simulation data}

Fig. 4 shows a comparison of the performances of the ICA decompositions and the GLM on the simulated data sets at the four investigated ACLs of the injected simulated activation signal $(\mathrm{ACL}=0.5,1,1.5$, and $2 \%$ ). The ROC power ranging from the values of 0.3 and 0.5 corresponded 


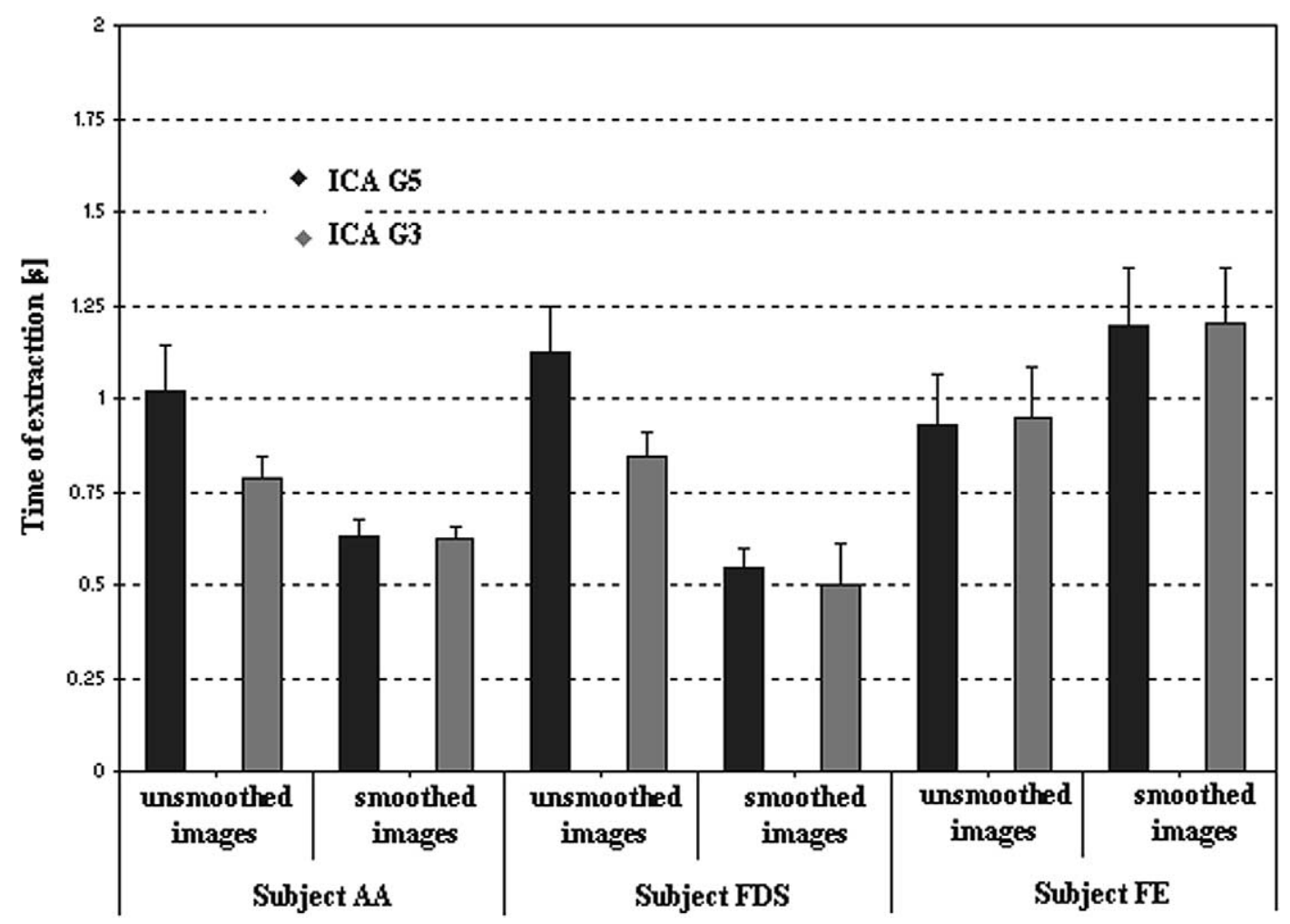

Fig. 3. Statistics on the elapsed times for the extraction of the task-related Independent Component in our case subjects $(1 \mathrm{slice}$, TR $=4 \mathrm{~s}$, Window $=10$, Sampling Factor $=1$, Step $=1)$. Two contrast functions $\left(G_{3}\right.$ and $\left.G_{5}\right)$ have been used.

to noisy although readable activation maps; over a value of 0.5 , the maps presented a remarkably good quality. ROC powers ranging from 0.1 to 0.3 corresponded to inadequate separation of the components.

Reported measures of spatial accuracy of the dynamic maps (Fig. 4a) revealed acceptable performances of ICA and GLM in the sliding-window mode at higher ACLs. Considering only the dynamic maps, the detection power of real-time methods falls at low ACLs but reaches acceptable levels of accuracy at high ACLs (ACLs $>1 \%$ ), thus covering a substantial part of the range of blood oxygen leveldependent (BOLD) contrasts achievable with 1.5-T scanners. ICA maps resulted slightly more accurate in terms of the average ROC power than GLM maps at high ACLs.

Different indications (Fig. 4b) may be drawn from the measure of spatial accuracy on the cumulative maps. In fact, considering only the cumulative maps and compared to the GLM applied in a cumulative mode, real-time ICA has provided acceptable results even at ACLs of 0.5 and $1 \%$. Both at low and at high ACLs the ROC time course of ICA was always comparable to, or even better than, that of the GLM, with and without linear detrending.

\section{Performances of real-time ICA on experimental data}

Figs. 5 and 6 show ROC results on real activation data. Preliminarily, we remark that the ROC power only mea- sures how accurately the activation maps generated in realtime reproduce the activity patterns resulting from the offline application of the same methods. We report ROC performances separately for the three subjects without (Fig. 5) and with (Fig. 6) the smoothing filter applied to the image time-series. In order to show the effects of different statistical properties of the data, mainly related to differences among individual signal and noise sources, these measures were not averaged across subjects. The values and time courses of the ROC power gave similar indications as those observed for the simulated data sets. Some intersubject differences were present, likely related to the variability of responsiveness and behavior of the subjects when considered on a scan-by-scan and trial-by-trial basis.

Spatial smoothing improved the detection power and reduced the intersubject differences of sliding-window realtime ICA results. The performances became, thus, more homogeneous across subjects (Fig. 6). Fig. 7 shows four consecutive frames from real-time ICA results obtained in slice 2 (of the four slices acquired). The visual inspection of the maps generated dynamically by the real-time decompositions, revealed a quality confirming the ROC measures. Specifically, both the dynamic and cumulative maps clearly show two main clusters of cortical activity corresponding to the main areas expected to be activated by the motor task: the primary motor cortex (PMC) and the supplementary motor area (SMA). 

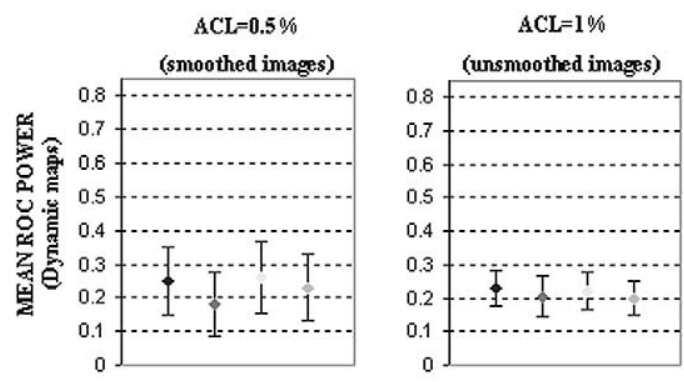

(a)
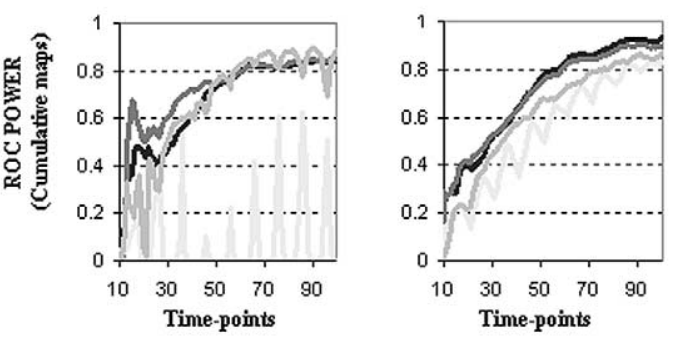

- ICA G5

$\rightarrow$ ICA G3

GLM D0

GLMDI

(b)

$\mathrm{ACL}=1.5 \%$
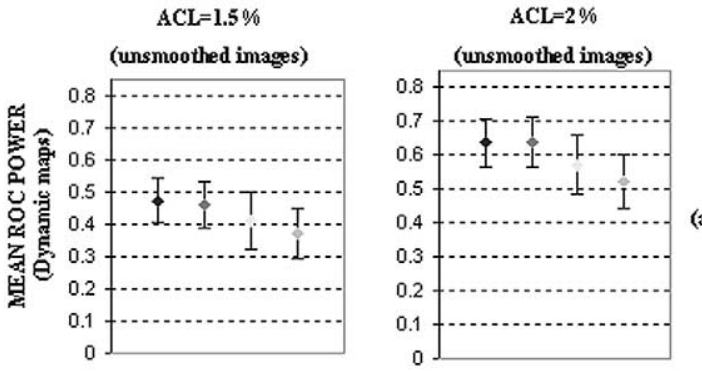

(a)

- ICA G5

- ICA G3

GLM Do
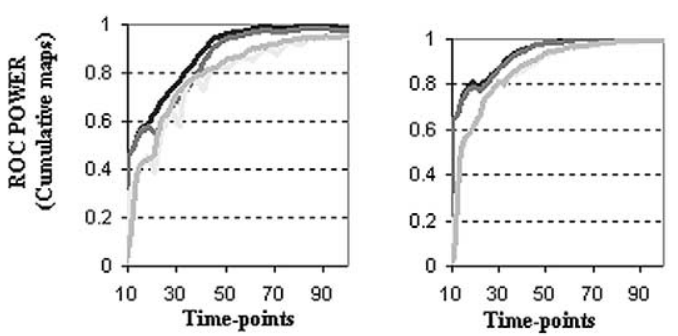

GLMDl

(b)

Fig. 4. Accuracy evaluation of results on simulated data. Comparisons between real-time ICA (with functions $G_{3}$ and $G_{5}$ ) and sliding-window GLM [without (GLM0) and with (GLM1) first order detrending]. (a) ROC statistics on dynamic readouts. (b) ROC time courses for the cumulative maps.

Four sequential time points $(41,46,51,56)$, separated from each other by a distance corresponding to the length of the blocks, were chosen for display and analysis of the dynamic decomposition. Specifically, the frames in Fig. 7 allow to appreciate the stability of the cumulative map and the successful tracking of the cumulative time course. These results are a direct consequence of the successful extraction and selection of the pretargeted motor components. Here, the simple ROI-based selection criterion was sufficient for the purpose of accurately selecting the task-related component. Consequently, the history of the task-related phenomena across the scans was easily traced without the use of temporal references, despite the dynamic target patterns (highlighted in green in Fig. 7) showed a clear scan-by-scan variability, particularly in the contribution of SMA and pre-SMA areas. Those variations are likely to be associated with the different amplitudes and delays of the responses of those nonprimary areas (Lee et al., 1999), whose voxels' contribution to the sliding-window covariance matrix varies across successive scans.

Even if the ROC powers estimated on the dynamic patterns in the quantitative analysis (Fig. 6) were dramatically affected by this temporal variability, resulting in more false positives and negatives, the cumulative maps progressively gained accuracy in ROC power, with the final pattern resembling more and more closely the off-line pattern, adopted as benchmark for the analysis.

Fig. 8 shows four real-time ICA decompositions from the four acquired slice time-series. Real-time ICA was run for each slice time-series separately, with identical temporal settings and highly similar elapsed times of extraction. The reported frames are fairly representative of typically observed features of real-time ICA readouts and shows qualitatively the capabilities of real-time ICA in representing various types of signals and artifacts. These sources may be grouped in task-related signals, non-task-related but function-related signal sources, motion-related artifacts, and vascular artifacts (McKeown et al., 1998b; Calhoun et al., 2003).

The task-related signals, were consistently detected with the used window of 10 scans, corresponding to the cycle of the paradigm. In our motor experiments, the ROI-based selection of the single task-related component was easily performed on the basis of the gyral anatomy because the activation focus in the primary motor cortex was highly stationary in space (see the maps highlighted in green in Figs. 7 and 8 ).

Function-related signals were identified on the basis of their spatial layout. These activities did not follow closely the stimulation paradigm, but their morphology and spatial locations were highly suggestive of brain functions not required by the task but likely to accompany inconstantly the main activity of the motor circuitry. The frontal and parietal eye-fields are an example of these components. They were recognizable as bilateral and symmetrical activation foci in the parietal and frontal cortices and were associated to time-courses weakly related to task block transition (Konishi et al., 2001). For instance, these components are visible in slices 2 and 4 in Fig. 8 (respectively IC5 and IC 3 in the blue boxes).

From a more general perspective, real-time ICA appears to be capable of detecting and translating signals resulting from different brain activities into readable activation maps. The possibility of detecting neural activities with unpredictable temporal behavior has already proved crucial, in an off-line setting, to elucidated complex, yet fundamental, mechanisms of brain physiology (Seifritz et al., 2002). Besides, the characterization of unpredictable phenomena, accompanying a task-related neural activity, may explain interactions and influences between the two activities taking 

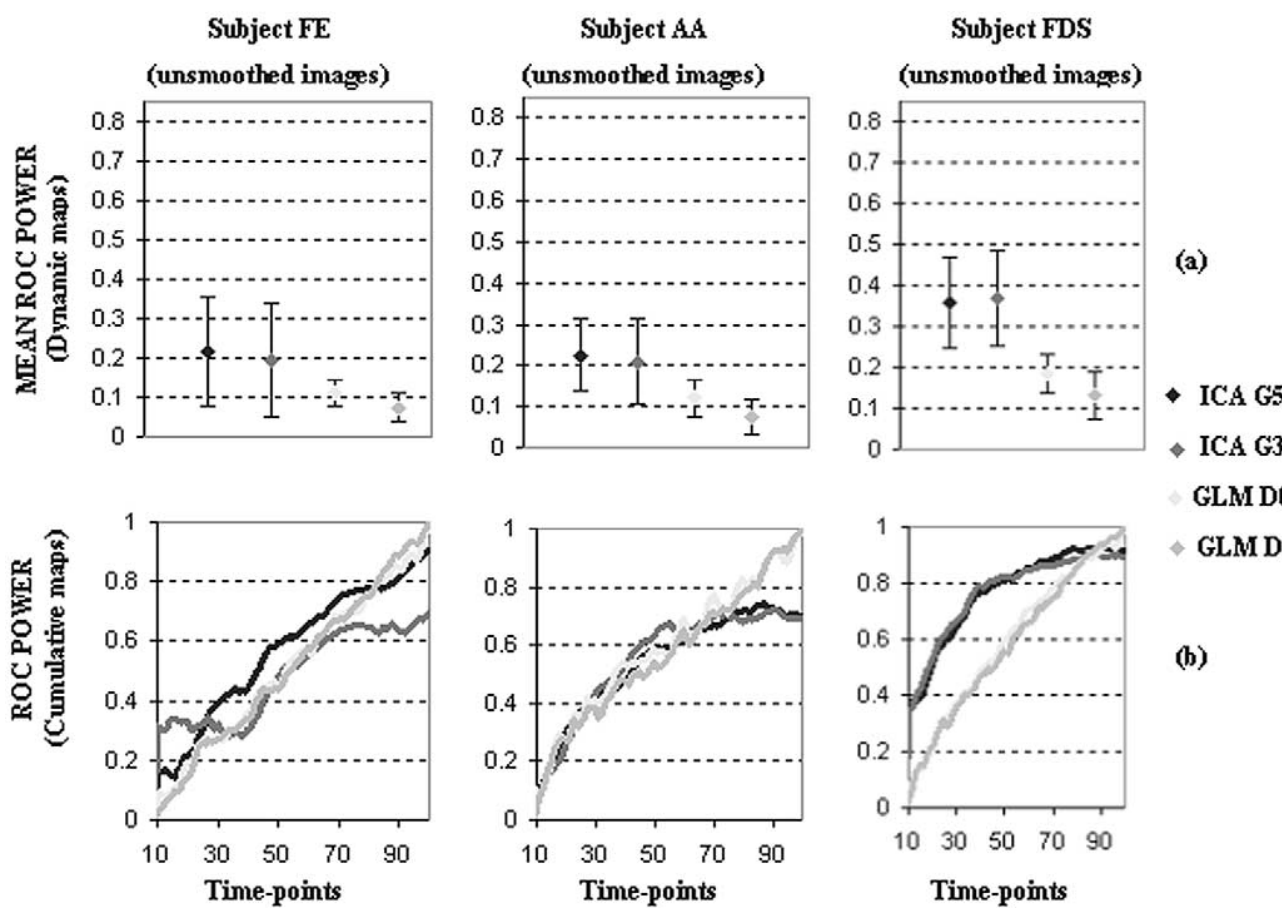

GLM D0

GLM DI

(b)

Fig. 5. Accuracy evaluation of results on real motor activation data (unsmoothed images). Comparisons between real-time ICA and sliding-window GLM [without (GLM0) and with (GLM1) first-order detrending]. (a) ROC statistics on dynamic readouts. (b) ROC time courses for the cumulative maps.

place at a cognitive level or at the level of local hemodynamics. In those situations, real-time ICA would really operate as a tool for a data-driven real-time control of behavioral states and task performances. In other words, it would provide not only the direct monitoring of the expected evoked signals, but even the additional insight of the neural phenomena underlying possible unintended changes in brain activity.
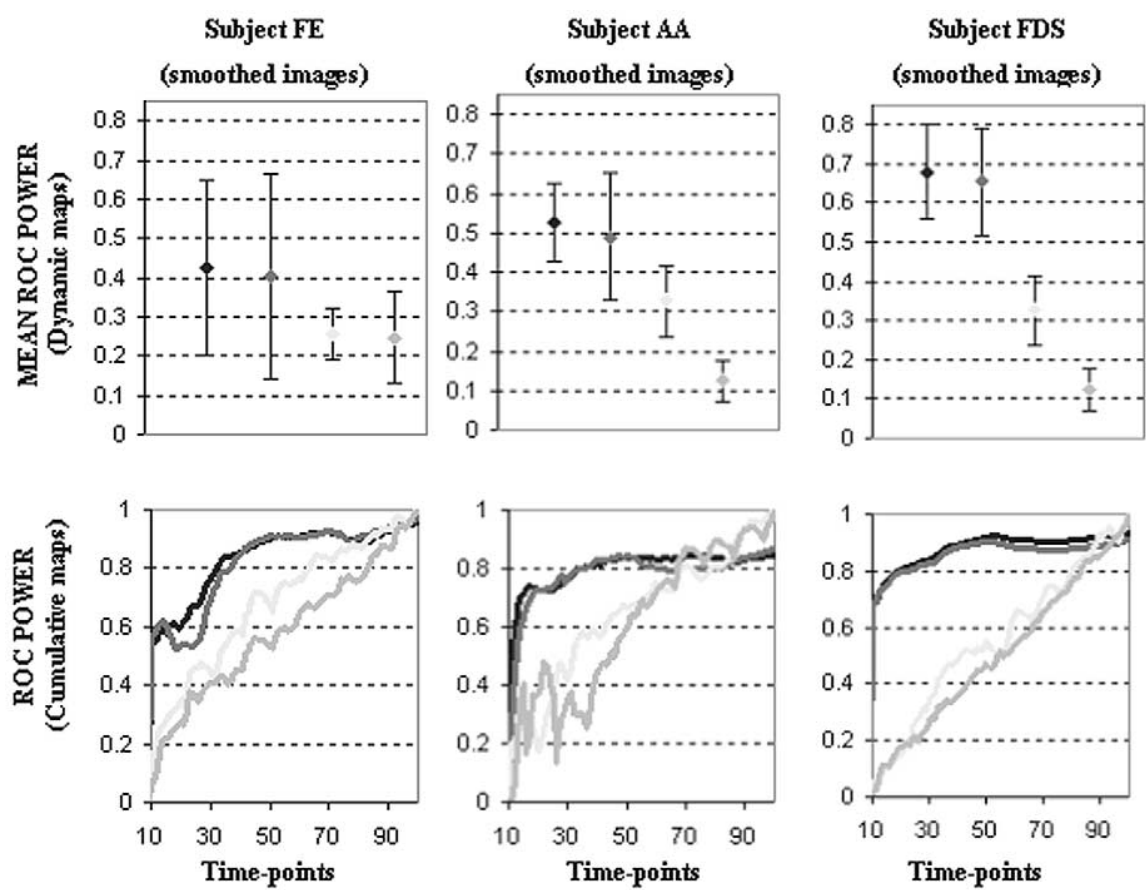

(a)

- ICA G5

- ICA G3

GLM DO
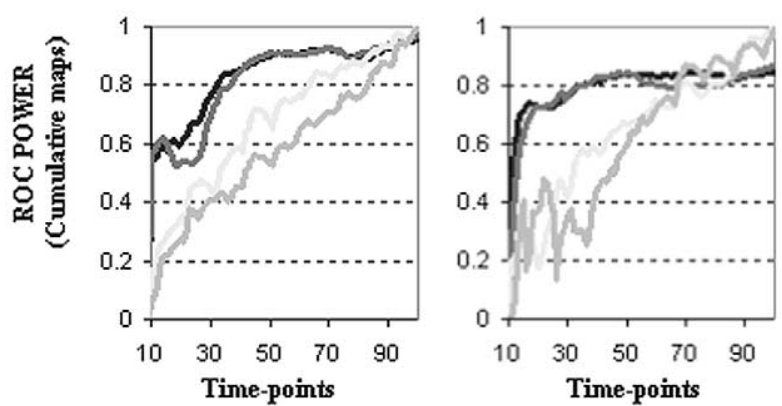

GLM D1

(b)

Fig. 6. Accuracy evaluation of results on real motor activation data (smoothed images). Comparisons between real-time ICA and sliding-window GLM [without (GLM0) and with (GLM1) first order detrending]. (a) ROC statistics on dynamic readouts. (b) ROC time-courses for the cumulative maps. 


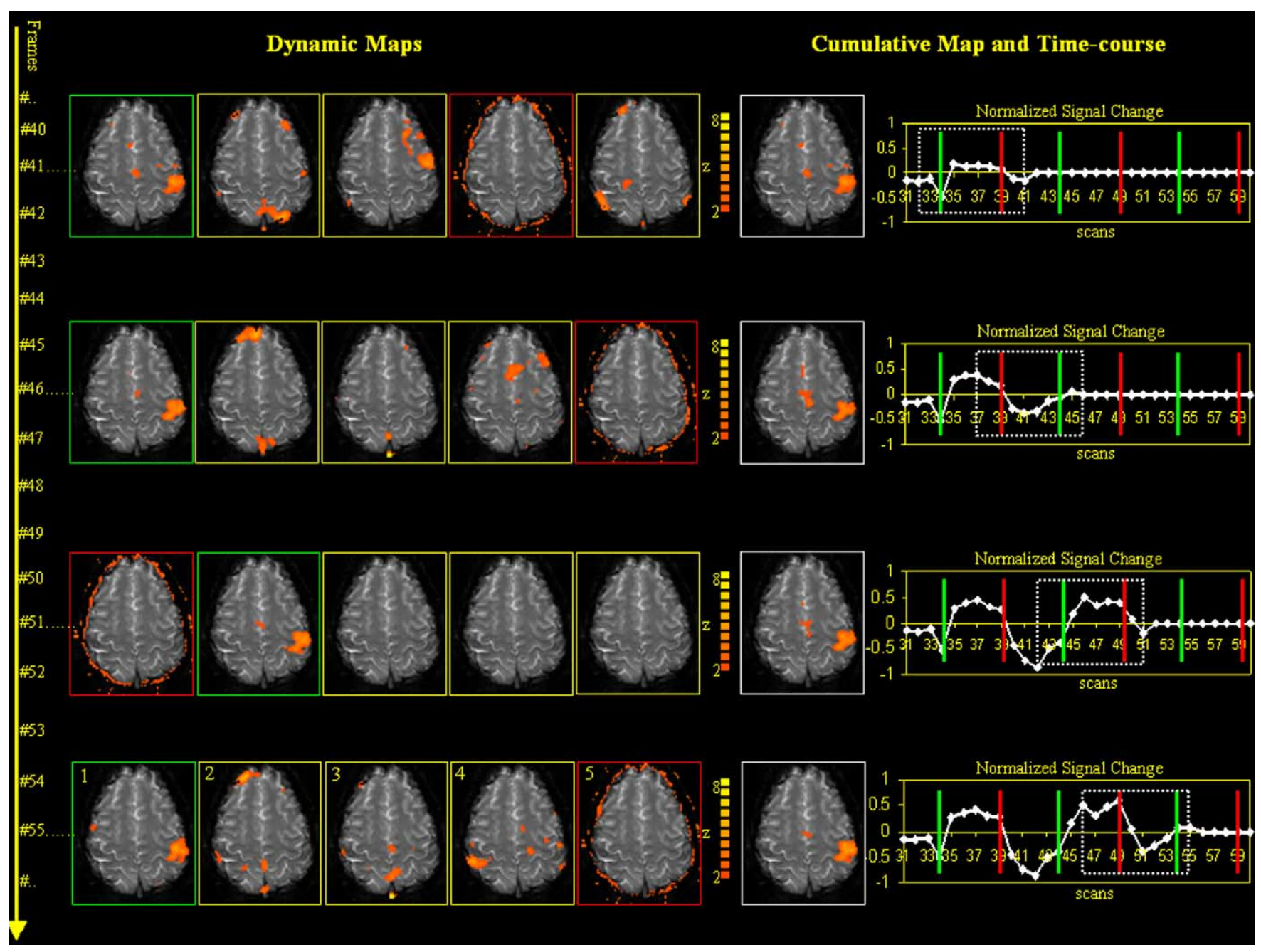

Fig. 7. Example of output of real-time ICA: dynamic maps (yellow, red, and green boxes) and cumulative maps (white boxes) and time-courses from five consecutive frames. The lower $(z=2)$ and upper $(z=8)$ thresholds of the maps are reported near the corresponding color scale. The maps in the green boxes correspond to the selected (here task-related ICs). The maps in the red boxes correspond to motion-related ICs.

In practice, during a typical fMRI session, the researcher may be promptly advised of different activities or alternative cognitive strategies performed by the subject, by just noting recurrent patterns in temporal coincidence with the expected activity. Motion-related signals were also frequent, causing dynamic components active at the edges of images. In previous articles, it was empirically demonstrated that spatial ICA applied to functional MRI timeseries is consistently able to separate, in one or more ringlike activation components, phenomena that are representative of head motion. Either long-term (slow) or abrupt signal change caused by the subject's head motion are consistently isolated by typical ICA-based methods (McKeown et al., 1998b). Apart from providing additional information about the quality of the raw time-series, this also results in partially motion-free task-related activation maps provided by other components of the decomposition.

It is widely known and accepted that head motion corrupts the signal changes induced by brain activation in
fMRI. A number of on-line (Mathiak and Posse, 2001) and off-line (Friston et al., 1996b) image registration algorithms have been proposed, which allow the automatic detection of motion and the correction of its effects in the time-series (realignment). These techniques require a particular transformation of the images with some form of interpolation of the pixel intensity. Although this allows to recover the task-related activated areas, the pixel values are inevitably modified in their time course by the interpolation-based reslicing of the same images. It has been shown (Grootoonk et al., 2000; Freire and Mangin, 2001) that this procedure may affect the precision of fMRI analyses, producing some false-positive results, if the interpolation scheme is not adequately tailored to the acquired data. From this perspective, a method that estimates motion-related sources of signal change in the acquired time-series may prove to be advantageous in all those situations where conventional model-based motion-compensation techniques do not work properly. Off-line ICA detects gradual and sudden motion 

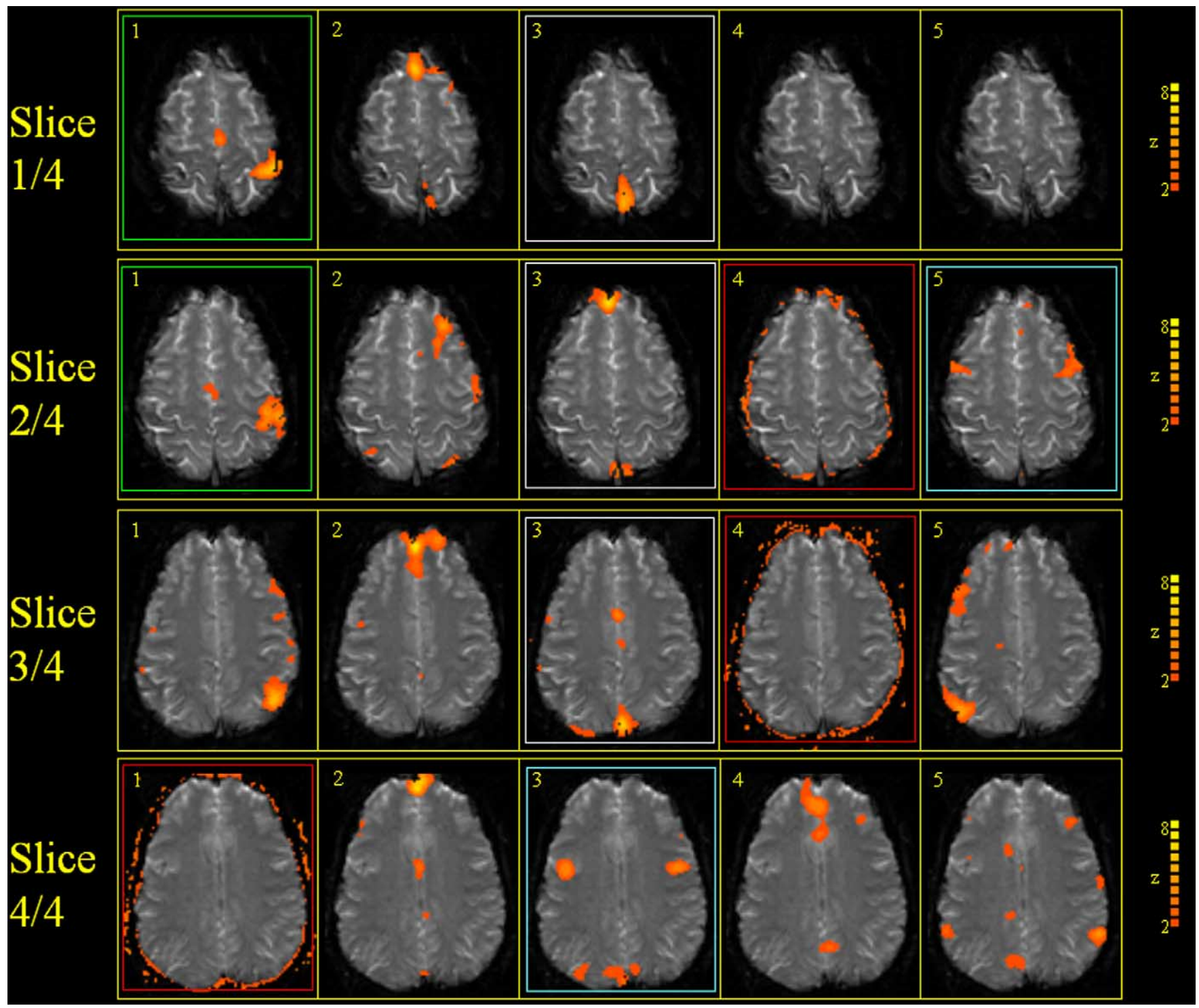

Fig. 8. Example of output of real-time ICA: dynamic maps from four different slices. The lower $(z=2)$ and upper $(z=8)$ of the maps are reported near the corresponding color scale. The maps in the green boxes correspond to the task-related ICs. The maps in the red boxes correspond to motion-related ICs. The maps in the blue boxes correspond to function-related ICs (parietal and frontal eye fields). The maps in the white boxes correspond to vascular artifacts.

without a preliminary correction of the data for the subjects' confined head motion (McKeown et al., 1998b). Thus, although the output patterns produced by the ICA decomposition are not expected to be equivalent with or without a compensation of the motion by a motion correction algorithm, task-related components generated by ICA are conceptually robust to motion effects.

Here we observe that, even in its real-time formulation, ICA preserves the capability to recognize in one or more components motion-related signal changes in the data and provides, in the form of ringlike patterns, a real-time feedback of subjects' movements during an ongoing session (in Fig. 7 and 8 the motion-related components are showed in the red boxes). Vascular artifacts (Dagli et al., 1999) are BOLD-related components showing activation foci in the regions of large blood vessels (Gu et al., 2001; Carroll et al.
2002). These components have often been extracted by real-time ICA in our data. For instance, the patterns shown in the white boxes of Fig. 8 (IC3 in slices 1, 2, and 3) belong to this class, because a focal activity matches anatomically a large vessel, as identified on the same raw EPI scan.

\section{Conclusions}

Our study demonstrates the feasibility and discusses the potentialities of using ICA in monitoring brain activity in a real-time setting. We have experimentally compared a fixed-point ICA algorithm, applied in sliding-window fashion, to standard linear regression-based real-time fMRI analysis methods, in either sliding-window and cumulative approach, with and without a first order (linear) trend re- 
moval. The quantitative comparison was restricted to the estimation of task-related signals, corresponding to one of four categories of the signals and artifacts that we have found to be regularly extracted by real-time ICA.

For the task-related signals, we present a standard ROC analysis quantifying the performances of real-time ICA in terms of accuracy and comparing them to sliding window and cumulative linear regression analysis on simulated and real activation time-series. Off-line generated patterns of activation were used as benchmarks in this analysis. We also exploited the potential of real-time ICA to detect and separate non-task-related, and even unexpected and shortlasting, changes in the data, as in the case of unexpected neural activities or of vascular and motion artifacts (that typically occurred in few successive positions of the sliding window).

The possibility of monitoring any kind of neural activities, together with the task-related effects, offers enlarged and intriguing opportunities for all those studies for which real-time analysis has been wished and proposed (Weiskopf et al., 2003).

\section{Acknowledgment}

Study was supported by Swiss National Science Foundation Grant 63-58040.99.

\section{References}

Andersen, A.H., Gash, D.M., Avison, M.J., 1999. Principal component analysis of the dynamic response measured by fMRI: a generalized linear systems framework. Magn. Reson. Imag. 17 (6), 795-815.

Bandettini, P.A., Wong, E.C., Hinks, R.S., Tikofsky, R.S., Hyde, J.S., 1992. Time course EPI of human brain function during task activation. Magn. Reson. Med. 25, 390-397.

Bandettini, P.A., Jesmanowicz, A., Wong, E.C., Hyde, J.S., 1993. Processing strategies for time-course data sets in functional MRI of the human brain. Mag. Reson. Med. 30, 131-176.

Bell, A.J., Sejnowski, T.J., 1995. An Information-Maximisation approach to blind separation and blind deconvolution. Neural Comput. 7, 10041034.

Brown, G.D., Yamada, S., Sejnowski, T.J., 2001. Independent Component Analysis at neural cocktail party. Trends Neurosci. 24 (1), 54-63.

Bullmore, E.T., Rabe-Hasketh, S., Morris, R.G., Williams, S.C.R., Gregory, L., Gray, J.A., Brammer, M.J., 1996. Functional magnetic resonance image analysis of a large-scale neurocognitive network. NeuroImage 4 (1), 16-33.

Calhoun, V.D., Adali, T., Pearlson, G.D., Pekar, J.J., 2001. Spatial and temporal independent component analysis of functional MRI data containing a pair of task-related waveforms. Hum. Brain. Mapp. 13, 43-53.

Calhoun, V.D., Adali, T., Pearlson, G.D., 2001. Independent component analysis applied to fMRI data: a generative model for validating results, in: Proc. IEEE Workshop on Neural Networks for Signal Processing (NNSP), Falmouth, MA, pp. 509-518. J. VLSI Sign. Process. Syst. Sign. Imag. Vid. Technol. (in press).

Carroll, T.J., Haughton, V.M., Rowley, H.A., Cordes, D., 2002. Confounding effect of large vessels on MR perfusion images analysed with independent component analysis. AJNR Am. J. Neuroradiol. 23, 10071012.
Castelo-Branco, M., Formisano, E., Backes, W., Zanella, F., Neuenschwander, S., Singer, W., Goebel, R., 2002. Activity patterns in human motion-sensitive areas depend on the interpretation of global motion. Proc. Natl. Acad. Sci. USA 1599 (21), 13914-13919.

Comon, P., 1994. Independent Component Analysis, a new concept? Sig. Proc. 36, 287-314.

Cox, R.W., Jesmanowicz, A., Hyde, J.S., 1995. Real-time functional magnetic resonance imaging. Magn. Reson. Med. 33, 230-236.

Dagli, M.S., Ingeholm, J.E., Haxby, J.V., 1999. Localization of cardiacinduced signal change in fMRI. NeuroImage 9, 407-415.

Duann, J.R., Jung, T.P., Kuo, W.J., Yeh, T.C., Makeig, S., Hsieh, J.C., Sejnowski, T.J., 2002. Single-trial variability in event-related BOLD signals. NeuroImage 15, 823-835.

Esposito, F., Formisano, E., Seifritz, E., Goebel, R., Morrone, R., Tedeschi, G., Di Salle, F., 2002. Spatial independent component analysis of functional MRI time-series: to what extent do results depend on the algorithm used? Hum. Brain Mapp. 16 (3), 146-157.

Everitt, B.S., Bullmore, E.T., 1999. Mixture model mapping of brain activation in functional magnetic resonance images. Hum. Brain Mapp. 7, 1-14.

Fadili, M.J., Ruan, S., Bloyet, D., Mazoyer, B., 2000. A multistep unsupervised fuzzy clustering analysis of fMRI time series. Hum. Brain Mapp. 10, 160-178.

Formisano, E., Esposito, F., Kriegeskorte, N., Tedeschi, G., Di Salle, F., Goebel, R., 2002. Spatial independent component analysis of functional magnetic resonance imaging time-series: characterization of the cortical components. Neurocomputing 49 (1-4), 241-254.

Freire, L., Mangin, J.F., 2001. Motion correction algorithms may create spurious brain activations in the absence of subject motion. NeuroImage 14 (3), 709-22.

Friedman, J.H., 1986. Exploratory projection pursuit. J. Am. Statist. Assoc. 82 (397), 249-266.

Friston, K.J., Frith, C., Liddle, P., Frackowiak, R.S.J., 1993. Functional connectivity: the principal component analysis of large data sets. J. Cereb. Blood Flow Metab. 13, 5-14.

Friston, K.J., Holmes, A.P., Worsley, K.J., Poline, J.P., Frith, C.D., Frackowiak, R.S.J., 1995a. Statistical parametric maps in functional imaging: a general linear approach. Hum. Brain Mapp. 2, 189-210.

Friston, K.J., Frith, C.D., Frackowiak, R.S., Turner, R., 1995b. Characterizing dynamic brain responses with fMRI: a multivariate approach. Neuroimage 2 (2), 166-72.

Friston, K.J., Williams, S., Howard, R., Frackowiak, R.S., Turner, R., 1996. Movement-related effects in fMRI time-series. Magn. Reson. Med. 35 (3), 346-355.

Gembris, D., Taylor, J.G., Schor, S., Frings, W., Suter, D., Posse, S., 2000. Functional MR Imaging in Real-Time using a sliding-window correlation technique. Magn. Reson. Med. 43, 259-268.

Grootoonk, S., Hutton, C., Ashburner, J., Howseman, A.M., Josephs, O., Rees, G., Friston, K.J., Turner, R., 2000. Characterization and correction of interpolation effects in the realignment of fMRI time series. NeuroImage 11, 49-57.

Gu, H., Engelien, W., Feng, H., Silbersweig, D.A., Stern, E., Yang, Y., 2001. Mapping transient, randomly occurring neuropsychological events using independent component analysis. Neuroimage 14, 14321443.

Hyvärinen, A., 1998. New approximations of differential entropy for independent component analysis and projection pursuit. Adv. Neural Inf. Proc. Syst. 10, 273-279.

Hyvärinen, A., 1999. Fast and robust fixed-point algorithms for independent component analysis. IEEE Trans. Neural Networks 10 (3), 626634.

Konishi, S., Donaldson, D.I., Buckner, R.L., 2001. Transient activation during block transition. NeuroImage 3 (2), 364-374.

Kwong, K.K., 1995. Functional magnetic resonance imaging with echo planar imaging. Magn. Reson. Q. 11, 1-20. 
Lee, K.M., Chang, K.H., Roh, J.K., 1999. Subregions within the supplementary motor area activated at different stages of movement preparation and execution. NeuroImage 9 (1), 117-123.

Mathiak, K., Posse, S., 2001. Evaluation of motion and realignment for functional magnetic resonance imaging in real-time. Magn. Reson. Med. 45 (1), 167-171.

McKeown, M.J., Jung, T.P., Makeig, S., Brown, G., Kindermann, S.S., Lee, T.W., Sejnowski, T.J., 1998a. Spatially independent activity patterns in functional MRI data during the stroop color-naming task. Proc. Natl. Acad. Sci. USA 95 (3), 803-810.

McKeown, M.J., Makeig, S., Brown, G.G., Jung, T.P., Kindermann, S.S., Bell, A.J., Sejnowski, T.J., 1998b. Analysis of fMRI data by blind separation into spatial independent component analysis. Hum. Brain Mapp. 6 (3), 160-188.

Mitra, P.P., Pesaran, B., 1999. Analysis of dynamic brain imaging data. Biophys. J. 76 (2), 691-708.

Papoulis, A., 1991. Probability, Random Variables, and Stochastic Processes. McGraw-Hill, New York.

Posse, S., Binkofski, F., Schneider, F., Gembris, D., Frings, W., Habel, U. Salloum, J.B., Mathiak, K., Wiese, S., Kiselev, V., Graf, T., Elghahwagi, B., Grosse-Ruyken, M.-L., Eickermann, T., 2001. A new approach to measure single-event related brain activity using real-time fMRI: feasibility of sensory, motor, and higher cognitive tasks. Hum. Brain Mapp. 12, 25-41.
Seifritz, E., Esposito, F., Hennel, F., Mustovic, H., Neuhoff, J.G., Bilecen, D., Tedeschi, G., Scheffler, K., Di Salle, F., 2002. Spatiotemporal pattern of neural processing in the human auditory cortex. Science 297 (5587), 1706-1708.

Sychra, J.J., Bandettini, P.A., Bhattacharya, N., Lin, Q., 1994. Synthetic images by subspace transforms. I. Principal components images and related filters. Med. Phys. 21 (2), 193-201.

Skudlarski, P., Constable, R.T., Gore, J.C., 1999. ROC Analysis of statistical methods used in functional MRI: individual subjects. NeuroImage 9, 311-329.

Suzuki, K., Kiryu, T., Nakada, T., 2002. Fast and precise independent component analysis for high field fMRI time series tailored using prior information on spatiotemporal structure. Hum. Brain Mapp. 15 (1), 54-66.

Voyvodic, J.T., 1999. Real-time fMRI paradigm control, physiology, and behavior combined with near real-time statistical analysis. NeuroImage 10, 91-106

Weiskopf, N., Veit, R., Erb, M., Mathiak, K., Grodd, W., Goebel, R., Birbaumer, N., 2003. Physiological self-regulation of regional brain activity using real-time functional magnetic resonance imaging (fMRI): methodology and exemplary data. NeuroImage 19, 577-586.

Yoo, S.S., Guttmann, C.R.G., Zhao, L., Panych, L.P., 1999. Real-time adaptive functional MRI. NeuroImage 10, 596-606. 